\title{
Confined Jet Mixing in the Entrance of a Tubular Reactor
}

\author{
WARREN D. SEIDER and STUART W. CHURCHILL \\ University of Michigan, Ann Arbor, Michigan
}

\begin{abstract}
The equations for the conservation of momentum and mass were solved numerically for the region downstream from the exit of an axial jet into a confined annular stream of the same fluid. An implicit, alternating direction method was used. Numerical results were obtained for radius ratios of $0.281,0.470$, and 0.563 over a range of jet-to-annular velocities from 0.85 to 2.77 . The values for the limiting case of the entrance region in pipe flow are in good agreement with the values of Christiansen and Lemmon and Ventras, Duda, and Bargeron. Solution of the equa. tions for the conservation of chemical components produced results for component transfer in agreement with the experimental compositions obtained by Wood for jet mixing. Representative calculations were carried out for a chemical reaction. The results indicate the efficiency of the mixing process for this type of reactor.

Experiments were carried out with water and dye to define the conditions under which the assumption of steady, laminar jet mixing is valid. The experiments also indicated the conditions and location of turbulent breakup of the jet.
\end{abstract}

The results reported herein are part of a continuing research program on the application of finite-difference techniques for the solution of the partial differential equations, boundary conditions, and initial conditions representing transport processes. Hellums and Churchill $(1,2)$ and Wilkes and Churchill (3) developed solutions for stable, transient natural convection in a confined fluid. Samuels and Churchill (4) extended this technique to investigate the dynamic stability of a confined fluid heated from below.

In this investigation solutions are developed for momentum transfer, component transfer, and chemical conversion in a region of confined jet mixing. One stream in fully developed laminar flow enters this mixing region from the exit of a central pipe; a second miscible stream, also in fully developed laminar flow, enters from the annulus formed by an outer concentric pipe as illustrated in Figure 1. In the most general case changes in temperature and composition would influence the fluid mechanics in the mixing region through changes in physical properties. However, in this investigation uniform temperature and uniform fluid properties are postulated corresponding to a reaction between tracer quantities of solute in two streams of common solvent. For this limiting condition the equations for the conservation of mass and momentum are uncoupled from those for the conservation of components and can be solved separately. The equations for the conservation of components can then be solved in turn.

A wake is formed at the intersection of the two streams owing to the drag of the inner and outer walls of the central pipe. This wake is eventually dissipated by momentum transfer, and the velocity profile for fully developed laminar flow in the outer pipe is finally attained. Because of the wake, the behavior in the mixing region may be dynamically unstable, that is, more than one flow pattern may be attained for the same dimensions, flow rates, and fluid properties, even though steady laminar flow exists at the inlet and outlet of the region. The analytical portion of this investigation postulates the existence of steady, twodimensional (laminar) flow. Experiments were carried out to define the conditions under which this postulate is valid.

Warren D. Seider and Stuart W. Churchill are at the University of Pennsylvania, Philadelphia, Pennsylvania.
The assumption of steady, two-dimensional flow limits mixing in the Lagrangian sense to molecular diffusion, although mixing in the Eulerian sense also occurs by radial flow.

The rate of conversion in the inlet region of any reactor with more than one feed stream depends on the rate of mixing as well as on the reaction rate constant. The results of this investigation can be used to compare the efficiency of a confined jet reactor with that of a hypothetical reactor with complete and instant mixing.

Examples of the applicability of a confined jet reactor are (1) the introduction of a corrosive reactant through the central tube, thus minimizing contact with the outer wall, and (2) laminar diffusion flames in tubes, thus avoiding flashback and a flame-holder.

Further details concerning this investigation are given in reference 5 .

\section{PRIOR WORK}

A model for turbulent confined jet mixing has been proposed by Curtet (6). However this model does not provide a sufficiently quantitative description of the fluid mechanics in the mixing region to allow the calculation of chemical conversion and component transfer.

Several recent papers have described the solution of the partial differential equations for laminar flow in simpler geometries and without chemical reaction or component transfer. Wang and Longwell (7) applied Allen's finitedifference scheme to obtain a numerical solution for the development of a parabolic velocity profile between two parallel planes. Vrentas, Duda, and Bargeron (8) applied a more general relaxation technique to obtain a numerical solution for the entrance region of a pipe. Paris and Whitaker (9) presented a finite-difference solution for the development of a single, plane Poiseuille flow from the merger of two, plane Poiseuille flows, initially separated by a thin plate. The accuracy of their results is uncertain since they did not demonstrate the convergence of their numerical calculations.

Burke and Schumann (10) predicted the shape of laminar diffusion flames in a confined jet mixing region with surprising success considering the assumption of isothermal, plug flow of both streams and an instantaneous reaction 
rate. Savage (11) has recently discussed modifications of this model to widen its range of applicability.

The existence of laminar flow in a confined jet mixing region at sufficiently low Reynolds numbers has been demonstrated by Wood (12) for the diffusion of ethylene into nitrogen. His data provide a critical test for the calculations of this investigation.

\section{MATHEMATIOAL MODEL}

As indicated above, the model postulates a steady, twodimensional motion and constant physical properties. The corresponding equations of motion after de-dimensionalization are

$$
\begin{gathered}
U_{r} \frac{\partial U_{z}}{\partial R}+U_{z} \frac{\partial U_{z}}{\partial Z}=-\frac{\partial P}{\partial Z}+\frac{1}{R} \frac{\partial}{\partial R}\left(R \frac{\partial U_{z}}{\partial R}\right)+\frac{\partial^{2} U_{z}}{\partial Z^{2}} \\
U_{r} \frac{\partial U_{r}}{\partial R}+U_{z} \frac{\partial U_{r}}{\partial Z}=-\frac{\partial P}{\partial R}+\frac{\partial}{\partial R}\left\{\frac{1}{R} \frac{\partial}{\partial R}\left(R U_{r}\right)\right\}+\frac{\partial^{2} U_{r}}{\partial Z^{2}} \\
\frac{1}{R} \frac{\partial}{\partial R}\left(R U_{r}\right)+\frac{\partial U_{z}}{\partial Z}=0
\end{gathered}
$$

The corresponding equations for component transfer with the chemical reaction, $a A+b B \longrightarrow$ products, and negligible axial diffusion are

$$
\begin{aligned}
U_{r} \frac{\partial C_{A}}{\partial R}+U_{z} \frac{\partial C_{A}}{\partial Z}+K_{A} C_{A}^{a} C_{B}^{b} & \\
& =\frac{1}{N_{S c_{A}}}\left(\frac{1}{R} \frac{\partial}{\partial R}\left(R \frac{\partial C_{A}}{\partial R}\right)\right) \\
U_{r} \frac{\partial C_{B}}{\partial R}+U_{z} \frac{\partial C_{B}}{\partial Z}+K_{B} C_{A}^{a} C_{B}^{b} & \\
& =\frac{1}{N_{S c_{B}}}\left(\frac{1}{R} \frac{\partial}{\partial R}\left(R \frac{\partial C_{B}}{\partial R}\right)\right)
\end{aligned}
$$

Both the central and the annular jet are postulated to have fully developed laminar flow profiles, that is, the model does not account for the distortion of the velocity profiles of the streams entering the mixing region by upstream diffusion of vorticity. The calculations were accordingly limited to Reynolds numbers greater than 100 since the distortion may be significant for lower flow rates $(8,9)$. The model does account for axial diffusion of vorticity in the mixing region where it may be significant. The boundary conditions are thus

$$
\begin{aligned}
& R=0 \quad U_{T}=\frac{\partial U_{z}}{\partial R}=\frac{\partial C_{A}}{\partial R}=\frac{\partial C_{B}}{\partial R}=0 \\
& R=1 \quad U_{r}=U_{z}=\frac{\partial C_{A}}{\partial R}=\frac{\partial C_{B}}{\partial R}=0 \\
& Z=0\left\{\begin{array}{ll}
0<R<\lambda & U_{r}=0, U_{z}=N_{R e_{b}}\left(1-\left(\frac{R}{\lambda}\right)^{2}\right), \\
& C_{A}=1, C_{B}=0 \\
\lambda<R<1 & U_{r}=0, U_{z}=\frac{N_{R e_{a}}}{\beta-\alpha}\left(1-R^{2}+\alpha \ln R\right), \\
& C_{A}=0, C_{B}=1 \\
Z \rightarrow \infty U_{r} \rightarrow 0 U_{z} \rightarrow N_{R e}\left[1-R^{2}\right]
\end{array}\right\}
\end{aligned}
$$

This formulation implies that the inner tube wall has a negligible thickness.

Equations (1), (2), and (3) may be reexpressed in terms of the stream function $\Psi$ and the radial vorticity $\zeta$ as follows:

$$
\begin{gathered}
U_{r}\left[\frac{\partial \zeta}{\partial R}-\frac{2 \zeta}{R}\right]+U_{z} \frac{\partial \zeta}{\partial Z}=\frac{\partial^{2} \zeta}{\partial R^{2}}-\frac{1}{R} \frac{\partial \zeta}{\partial R}+\frac{\partial^{2} \zeta}{\partial Z^{2}} \\
\zeta=\frac{\partial^{2} \Psi}{\partial R^{2}}-\frac{1}{R} \frac{\partial \Psi}{\partial R}+\frac{\partial^{2} \Psi}{\partial Z^{2}} \\
U_{z}=-\frac{1}{R} \frac{\partial \Psi}{\partial R}, \quad U_{r}=\frac{1}{R} \frac{\partial \Psi}{\partial Z}
\end{gathered}
$$

Equations (7) to (9) will be referred to as the vorticity, stream function, and velocity equations, respectively. The corresponding boundary conditions are

$$
\begin{aligned}
& R=0 \quad \zeta=0, \quad \Psi=\frac{N_{R e}}{4} \\
& R=1 \quad \zeta=\frac{\partial^{2} \Psi}{\partial R^{2}}, \quad \Psi=0 \\
& \int 0<R<\lambda \quad \zeta=2 N_{R e_{b}}\left(\frac{R}{\lambda}\right)^{2} \\
& \Psi=N_{R e_{b}}\left(\frac{\lambda^{2}-R^{2}}{2}-\frac{1}{\lambda^{2}}\left(\frac{\lambda^{4}}{4}-\frac{R^{4}}{4}\right)\right)+ \\
& \frac{N_{R e_{a}}}{\beta-\alpha}\left(\frac{1}{4}(1-\alpha)-\frac{\lambda^{2}}{2}\left(1-\frac{\lambda^{2}}{2}+\alpha \ln \lambda-\frac{\alpha}{2}\right)\right) \\
& \lambda<R<1 \quad \zeta=\frac{N e_{a}}{\beta-\alpha}\left(2 R^{2}-\alpha\right), \\
& \Psi=\frac{N_{R e_{a}}}{\beta-\alpha}\left(\frac{1}{4}(1-\alpha)-\frac{R^{2}}{2}\left(1-\frac{R^{2}}{2}+\alpha \ln R-\frac{\alpha}{2}\right)\right) \\
& Z \rightarrow \infty \quad \zeta \rightarrow 2 N_{R e} R^{2}, \quad \Psi=\frac{N_{R e}}{4}\left[1-R^{2}\left(2-R^{2}\right)\right]
\end{aligned}
$$




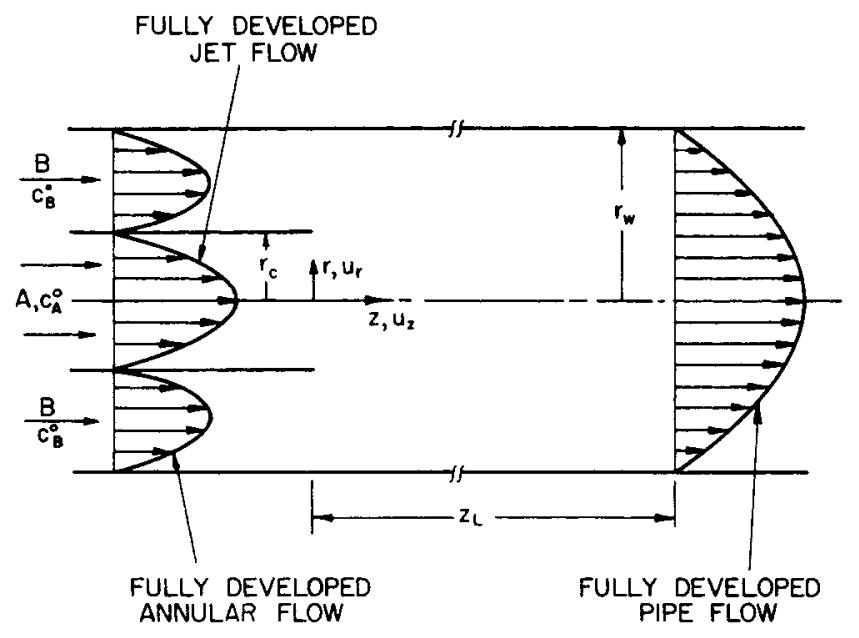

Fig. 1. Laminar, circular, confined jet mixing region.

Inspection of Equations (1), (2), (3), and (6) or (7) through (10) indicates that the velocity field is a function of only three parameters, $N_{R e_{b}}, N_{R e_{a}}$, and $\lambda$.

Equations (4) and (5) are coupled and require iterative solution. When it is justifiable to assume $D_{A S}=D_{B S}$, these equations can be rearranged as follows:

$$
\begin{aligned}
U_{r} \frac{\partial \Phi}{\partial R}+U_{z} \frac{\partial \Phi}{\partial Z}=\frac{1}{N_{S c}} & {\left[\frac{1}{R} \frac{\partial}{\partial R}\left(R \frac{\partial \Phi}{\partial R}\right)\right] } \\
U_{r} \frac{\partial C_{A}}{\partial R}+U_{z} \frac{\partial C_{A}}{\partial Z}+K_{A} C_{A}^{a} & {\left[\gamma_{2}\left(\frac{C_{A}}{\gamma_{2}}-\Phi\right)\right]^{b} } \\
& =\frac{1}{N_{S c}}\left[\frac{1}{R} \frac{\partial}{\partial R}\left(R \frac{\partial \Phi}{\partial R}\right)\right]
\end{aligned}
$$

with the following boundary conditions

$$
\begin{array}{cl}
R=0 & \frac{\partial \Phi}{\partial R}=\frac{\partial C_{A}}{\partial R}=0 \\
R=1 & \frac{\partial \Phi}{\partial R}=\frac{\partial C_{A}}{\partial R}=0 \\
Z=0\left\{\begin{array}{ll}
0<R<\lambda & \Phi=\frac{1}{\gamma_{1}}, C_{A}=1 \\
\lambda<R<1 & \Phi=-\frac{1}{\gamma_{2}}, C_{A}=0 .
\end{array}\right\}
\end{array}
$$

Equation (11) is simply Equation (12) for the nonreaction case $(k=0)$ and can be solved independently. The $\Phi$ field is substituted in Equation (12) to det ermine $C_{A}$, and $C_{B}$ is obtained from the definition of $\Phi$.

\section{FINITE-DIF FERENCE APPROXIMATION}

\section{Flow Field}

The partial differential equations can be approximated by finite-difference equations as follows. The confined jet mixing region is first subdivided by grid lines as indicated in Figure 2. The values of the dependent variables at each grid point (intersection of grid lines) are next approximated by series expansions in terms of the values of the dependent variables at the surrounding grid points. The derivatives are then replaced by values of the variables using the series expansions. This procedure yields an algebraic equation for each differential equation at each grid point. Alternatively these same algebraic equations can be derived directly by expressing the conservation of momentum, mass, etc., in the subregion around each grid point in terms of the values of the dependent variables at that grid point and at the surrounding grid positions, using a linear model for the several rate processes. The resulting set of algebraic equations must then be solved exactly or approximately.

The subdivision, the finite-difference representation, and the method of solution of the resulting equations are all arbitrary. The selection of a successful and efficient representation and method of solution usually requires both ingenuity and trial and error. Finally, the independence of the solution from these arbitrary choices must be demonstrated by changing the grid size and by comparing the numerical results with experimental data or with an analytical solution.

Equations (7) and (8) are elliptic. Equivalent equations have been solved successfully with modified relaxation methods ( 7 to 9). However numerical instability was encountered herein when these techniques were tried for the more complex boundary conditions corresponding to confined jet mixing.

The implicit, alternating-direction method can be shown to be unconditionally stable for the solution of linear, parabolic, partial differential equations. It was decided to try this method for the solution of the nonlinear equations in the hope that the nonlinearities would be insufficient in magnitude to generate numerical instability. First, Equation (7) was converted to a parabolic equation by adding a transient term:

$$
\frac{\partial \zeta}{\partial \tau}+U_{r}\left(\frac{\partial \zeta}{\partial R}-\frac{2 \zeta}{R}\right)+U_{z} \frac{\partial \zeta}{\partial Z}=\frac{\partial^{2} \zeta}{\partial R^{2}}-\frac{1}{R} \frac{\partial \zeta}{\partial R}+\frac{\partial^{2} \zeta}{\partial Z^{2}}
$$

Initial conditions were chosen by assuming the velocity profile to be that for fully developed laminar flow throughout the mixing region. At $\tau=0+$ the confined jet inlet condition was imposed. Although the resulting transient flow field does not have any practical significance, this technique produced a successful steady state solution.

With the implicit, alternating-direction method, the time step $\Delta \tau$ is split into two half-time steps. During the first half-step the vorticities are calculated implicitly in the $R$ direction using explicit, finite-difference approximations for the $Z$ derivatives; during the second half-step the vorticities are calculated implicitly in the $Z$ direction using explicit, finite-difference approximations for the $R$ derivatives. The corresponding equations are

$$
\begin{aligned}
\frac{\zeta_{i, j}^{*}-\zeta_{i, j}}{\frac{\Delta \tau}{2}}+U_{r_{i, j}}\left[\frac{\zeta_{i, j+1}^{*}-\zeta_{i, j-1}^{*}}{2 \cdot \Delta R}-\frac{2 \zeta_{i, j}^{*}}{j \cdot \Delta R}\right]+U_{z_{i, j}}\left[\frac{\zeta_{i_{+1, j}}-\zeta_{i-1, j}}{2 \cdot \Delta Z}\right]=\frac{\zeta_{i, j+1}^{*}-2 \zeta_{i, j}^{*}+\zeta_{i, j-1}^{*}}{\Delta R^{2}} \\
-\frac{1}{(j \cdot \Delta R)} \frac{\zeta_{i, j+1}^{*}-\zeta_{i, j-1}^{*}}{2 \cdot \Delta R}+\frac{\zeta_{i+1, j}-2 \zeta_{i, j}+\zeta_{i-1, j}}{\Delta Z^{2}}
\end{aligned}
$$


and

$$
\begin{aligned}
\frac{\zeta_{i, j}^{\prime}-\zeta_{i, j}^{*}}{\frac{\Delta \tau}{2}}+ & U_{r_{i, j}}\left[\frac{\zeta_{i, j+1}^{*}-\zeta_{i, j-1}^{*}}{2 \cdot \Delta R}-\frac{2 \zeta_{i, j}^{*}}{j \cdot \Delta R}\right]+U_{z_{i, j}}\left[\frac{\zeta_{i+1, j}-\zeta_{i-1, j}^{\prime}}{2 \cdot \Delta Z}\right]=\frac{\zeta_{i, j+1}^{*}-2 \zeta_{i, j}^{*}+\zeta_{i, j-1}^{*}}{\Delta R^{2}} \\
& -\frac{1}{(j \cdot \Delta R)} \frac{\zeta_{i, j+1}^{*}-\zeta_{i, j-1}^{*}}{2 \cdot \Delta R}+\frac{\zeta_{i+1, j}^{\prime}-2 \zeta_{i, j}^{\prime}+\zeta_{i-1, j}^{\prime}}{\Delta Z^{2}}
\end{aligned}
$$

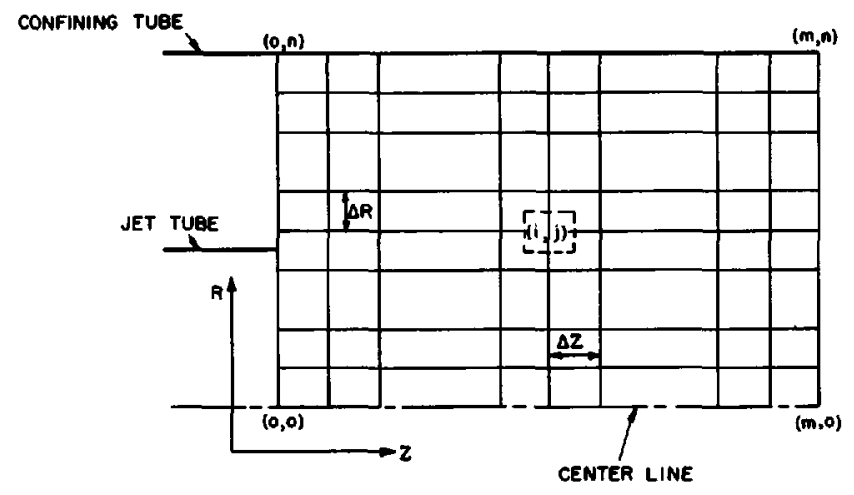

Fig. 2. Grid structure for confined jet mixing region.

During the first half-time step, in the $Z$ direction, along any row $i, \zeta_{i, j+1}^{*}, \zeta_{i, j}^{*}$, and $\zeta_{i, j-1}^{*}$ were implicitly calculated with $\zeta_{i+1, j}, \zeta_{i, j}, \zeta_{i-1, j}, U_{r_{i, j}}$, and $U_{z_{i, j}}$ held constant at values calculated at the end of the last time step. During the second half-time step, in the $R$ direction, along any column $j, \zeta_{i_{+}, j}^{\prime}, \zeta_{i, j^{\prime}}^{\prime}$ and $\zeta_{i-1, j}^{\prime}$ were implicitly calculated, with $\zeta_{i, j+1}^{*}, \zeta_{i, j}^{*}$, and $\zeta_{i, j-1}^{*}$ held constant at values calculated during the first half-time step, and $U_{r_{i, j}}, U_{z_{i, j}}$ held constant throughout the entire time step.

The solution began by calculating values of $\zeta$ at $\tau=\Delta \tau$ based on the known initial values of $\zeta, U_{z}$, and $U_{r}$. Corresponding values of $\Psi$ were calculated by numerical solution of Equation (8) using the just calculated values of $\zeta$ for $\tau=\Delta \tau$. The solution of Equation (8), which is also elliptic, was carried out by first adding a transient term, $\partial \Psi / \partial \theta$, thereby converting it to the following parabolic form:

$$
\frac{\partial \Psi}{\partial \theta}=-\zeta+\frac{\partial^{2} \Psi}{\partial R^{2}}-\frac{1}{R} \frac{\partial \Psi}{\partial R}+\frac{\partial^{2} \Psi}{\partial Z^{2}}
$$

The implicit, alternating-direction method was then used with the previous values of the stream-function field as initial values. This computation proceeded until the variation in the $\Psi$ field with time became negligible. The nature of Equation (17) permitted the use of very large "time steps," $\Delta \theta$, equal to 64 to 96 times $\Delta \tau$, resulting in a rapid rate of convergence. Generally only three $\Delta \theta$ steps were required.

The $U_{z}$ and $U_{r}$ fields and $\zeta_{w}$ were calculated using central-difference approximations and a two-point finitedifference approximation, respectively. Numerical instability was encountered when higher order approximations were used to calculate $\zeta_{w}$.

The downstream boundary $Z_{L}$ was initially chosen as $Z=20$, and was increased whenever the newly calculated values of $\zeta$ and $\Psi$ at $Z_{L}$ differed from the values for fully developed flow by more than $1.0 \%$. The entire procedure was carried out until a negligible change was achieved in values of $\zeta$ at $Z_{L}$ and $Z_{L}-\Delta Z$ and at $\tau$ and $\tau+\Delta \tau$.

\section{Chemical Reaction and Component Transfer}

Parabolic equations (11) and (12) with boundary conditions (13) were solved using the Crank-Nicolson finitedifference technique and the previously calculated values for the steady state velocity field. Equation (11) was solved first and then (12).

\section{NUMERICAL GALGULATIOHS}

The conditions chosen for illustrative calculations of confined jet mixing are summarized in Table 1. Laminar flow throughout the mixing region was observed experimentally for these conditions. The conditions of runs 1 and 3 correspond to the experiments of Wood (12). Calculations were also carried out for the development of a parabolic velocity profile from a uniform velocity profile in a tube at $N_{R e}=250$.

Calculations were carried out for the conditions of run 3 for a series of grid spacings with a fixed ratio $\Delta Z / \Delta R=20$ to establish convergence. The results are illustrated in Figure 3. The values for $\Delta R=0.05$ and $\Delta Z=1.0$ are within $6 \%$ of the extrapolated values for $\Delta R \rightarrow 0$. Since the computation time increased roughly as the cube of the number of grid points, no attempt was made to achieve greater accuracy and the reported results are for that grid spacing.

Instability was encountered in the vorticity calculation when $\Delta Z$ was reduced below 1.0 at $\Delta R=0.05$. The wake is apparently responsible for this restriction on $\Delta Z / \Delta R$ since the calculations for the entrance region of a tube were stable for $\Delta Z=0.25$ and $\Delta R=0.05$. With $\Delta R=0.05$ and $\Delta Z=1.0$, instability was encountered in the vorticity calculations at time steps $\Delta \tau$ greater than $6.25 \times 10^{-4}$. On the other hand, stream-function calculations were stable for $\Delta \theta$ as high as 0.06 .

Wood (12) reported the existence of stable laminar flows at jet-to-annular velocity ratios as high as 10.0. However numerical instability prevented calculations at velocity ratios $U_{z_{b}} / U_{z_{a}}$ greater than 4 with the grid sizes used herein. It is probable that the formulation would be stable up to a velocity ratio of 10.0 with a finer grid. The cost of the calculations would however become prohibitive. The challenge remains therefore to develop a still more stable procedure than described herein. Numerical instability was not encountered in the component transfer and reaction calculations.

The transient calculations were assumed to have converged to the steady state when vorticities, stream functions, and velocities all agreed within $0.1 \%$ in successive time steps $\Delta \tau$. Each run in Table 1 required nearly 30 min. on an IBM-7090 with $\Delta R=0,05$ and $\Delta Z=1.0$. This time was reduced to $4 \mathrm{~min}$. with $\Delta R=0.10$ and $\Delta Z=2$ with the related loss in accuracy indicated in Figure 3 .

Table 1. Confined Jet Flow Systems

\begin{tabular}{clccc}
\hline \hline Run No. & $\lambda$ & $N_{R e_{b}}$ & $N_{R e_{a}}$ & $N_{R e}$ \\
\hline 1 & 0.281 & 139 & 354 & 493 \\
2 & 0.47 & 294 & 119 & 313 \\
3 & 0.563 & 250 & 228 & 496 \\
\hline
\end{tabular}




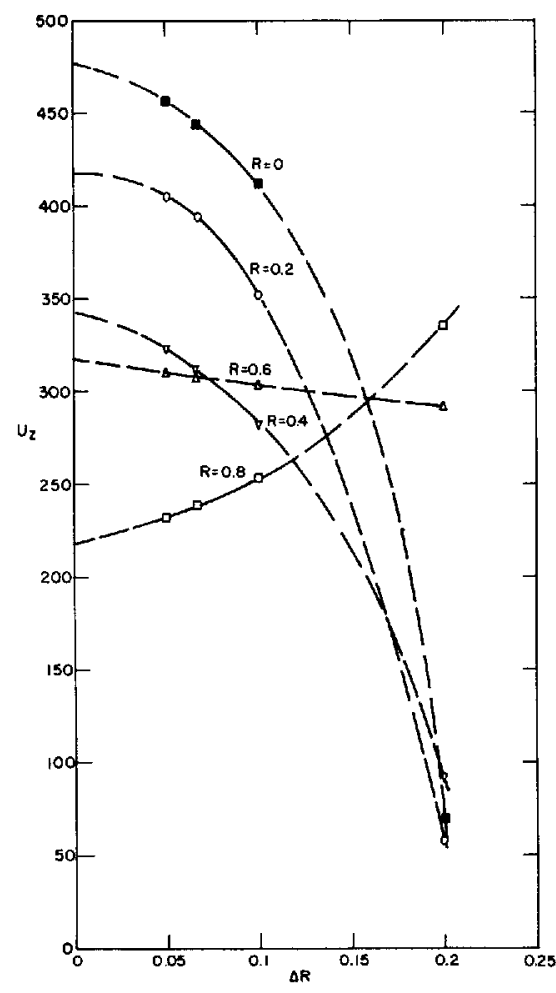

Fig. 3. The effect of grid spacing on numerical results. $R$ un $3, Z=4.0, \Delta Z / \Delta R=20$.

\section{NUMERICAL RESULTS}

\section{Flow field}

The numerical results obtained by solution of the vorticity, stream function, and velocity equations are illustrated in Figures 4 to 6 . The vorticity profiles demonstrate the gradual elimination of opposing rotational forces in the wake. Axial velocity profiles illustrate the development of flow in the wake, and the streamline patterns most clearly demonstrate the flow of material into the wake. The common interaction of the velocity curves at $R \cong 0.53$ for run 1 is presumably fortuitous and does not occur for the other runs. For the cases studied, the wake was essentially eliminated in the first five confining tube diameters. Radial flows from the jet and the annular stream poured fluid into the wake, which generally remained approximately centered at the jet tube radius. The streamline patterns show only slight spreading and contracting of the jet. These results were in good agreement with the dye tracer experiments described subsequently. However the small sinusoidal oscillations observed in the experiments did not show up in the numerical results, possibly because the model was two-dimensional.

Numerical results for development of a parabolic velocity profile from a uniform profile in a pipe are presented in Figure 7 . They compare very favorably with the numerical results of Christiansen and Lemmon (13) and Vrentas, Duda, and Bargeron (8). This comparison is a reassuring if not sufficient verification of the validity of the alternating, finite-difference algorithm for the solution of entrance flow problems.

\section{Component Transfer Without Reaction}

The equation for component transfer without chemical reaction $(k=0)$ was first solved. The calculated concentrations are compared with the measurements of Wood for an ethylene tracer in nitrogen (12) in Figures 8 and 9. Good agreement is apparent between the calculated and experimental concentrations. Curves for plug flow and fully developed parabolic flow are included in Figure $9 a$. For these simplified models, the inlet concentration of solute

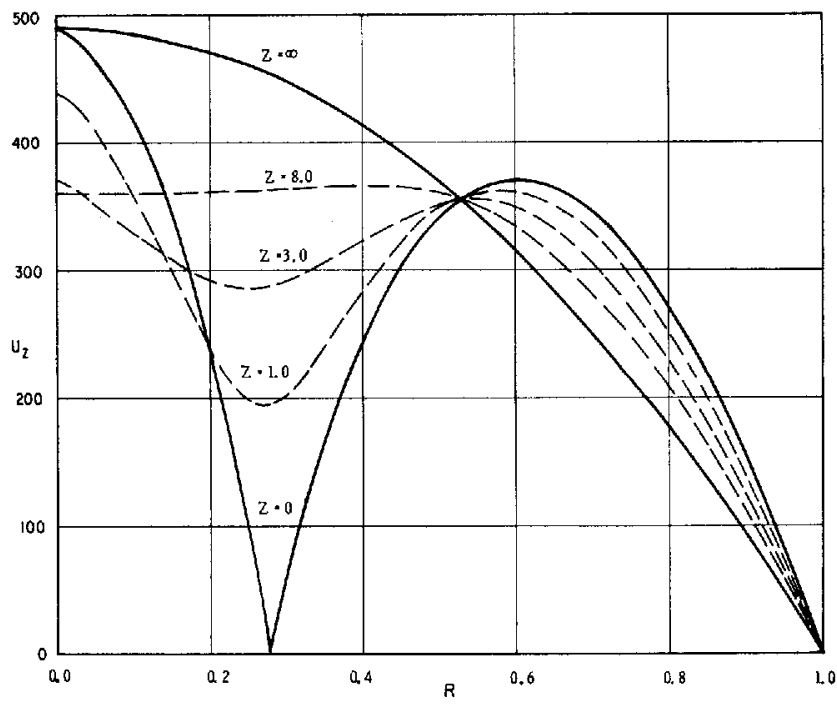

Fig. 4a. Confined jet velocity profiles. Run $1, \lambda=0.281$, $N_{R_{e}}=139, N_{R e_{a}}=354\left(N_{R e}=493\right)$.

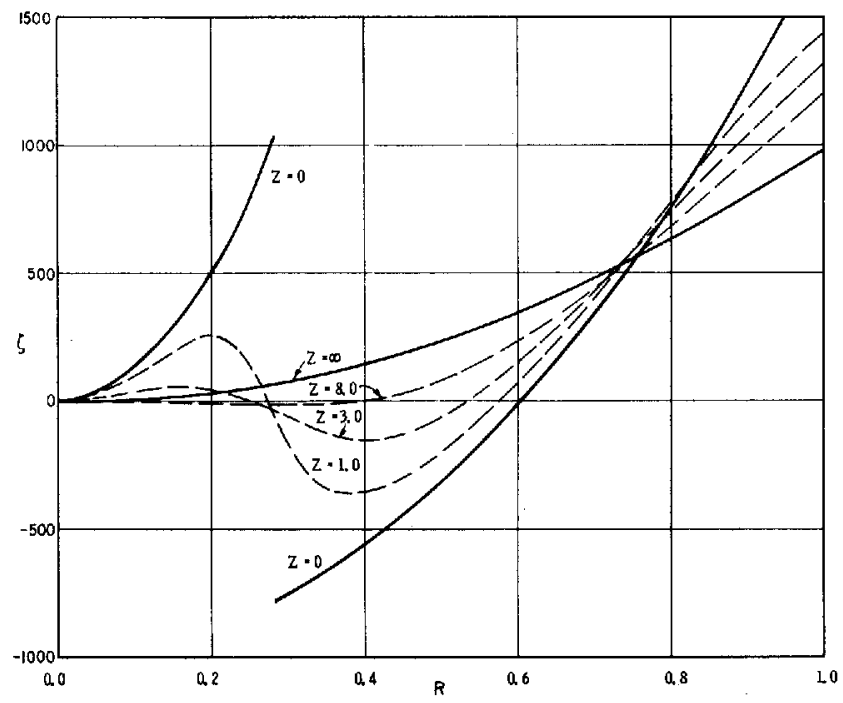

Fig, 4b. Confined jet vorticity profiles. Run $1, \lambda=0.281$, $N_{R_{b}}=139, N_{R e_{b}}=354\left(N_{R_{e}}=493\right)$.

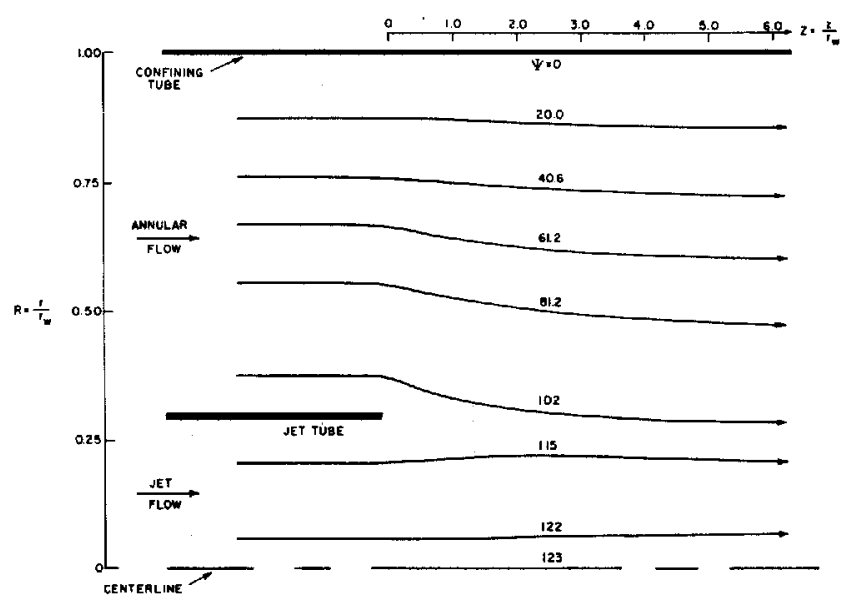

Fig. 4c. Confined iet streamlines. Run 1, $\lambda=0.281, N_{R e b}=139$, $N_{R_{\mathrm{e}}}=354\left(N_{R e}=493\right)$. 


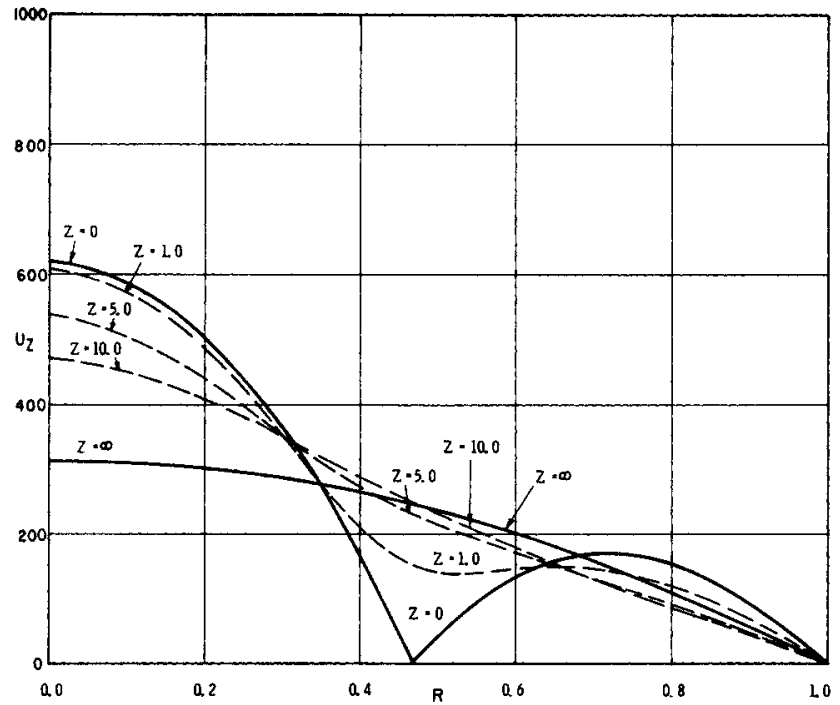

Fig. 5a. Confined jet velocity profiles. Run $2, \lambda=0.47, N_{R \in b}=$ 294, $N_{R_{e}}=119\left(N_{R e}=313\right)$.

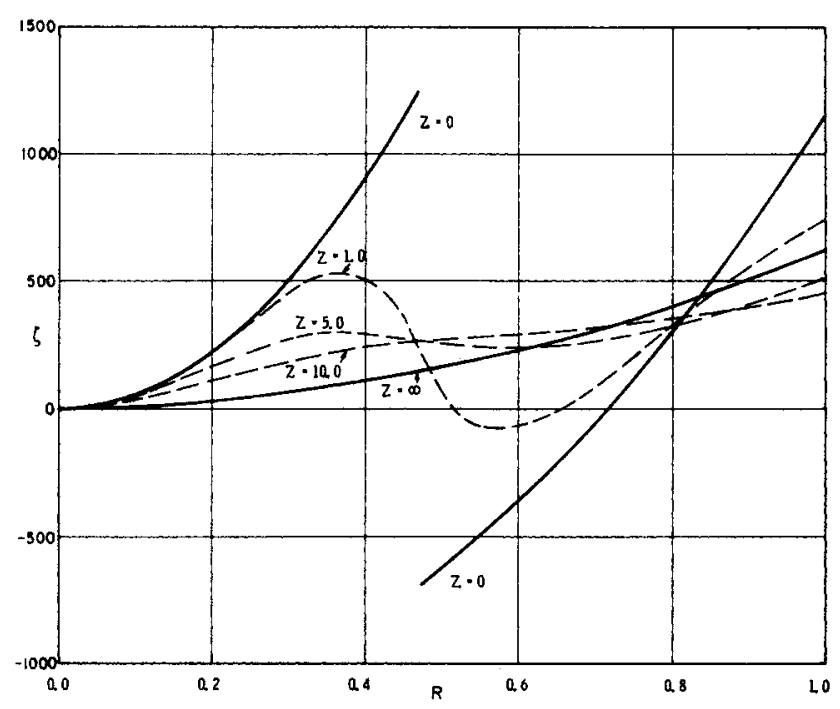

Fig. 5b. Confined jet vorticity profiles. Run $2, \lambda=0.47$, $N_{R e_{b}}=294, N_{R e_{a}}=119\left(N_{R e}=313\right)$.

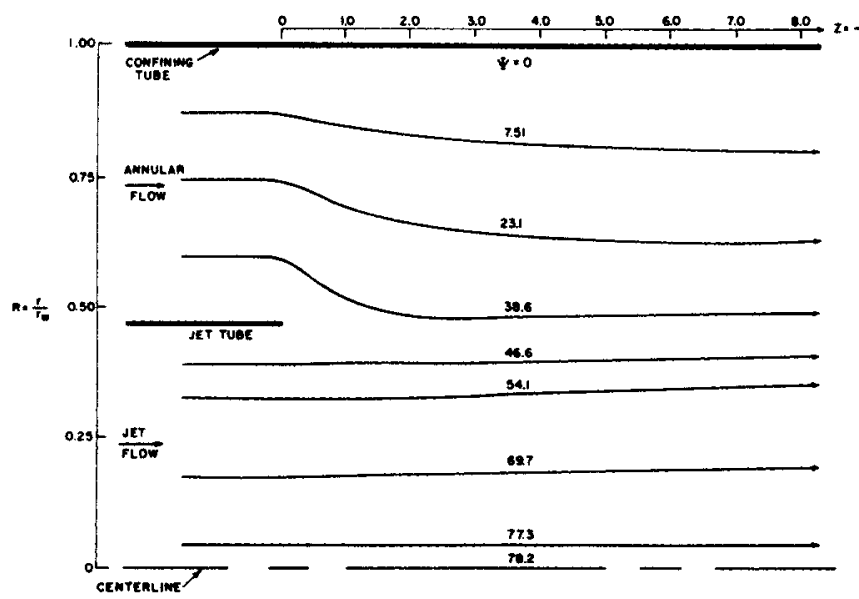

Fig. 5c. Confined jet streamlines. Run $2, \lambda=0.47, N_{R_{b}}=294$, $N_{R e_{0}}=119\left(N_{R e}=313\right)$.

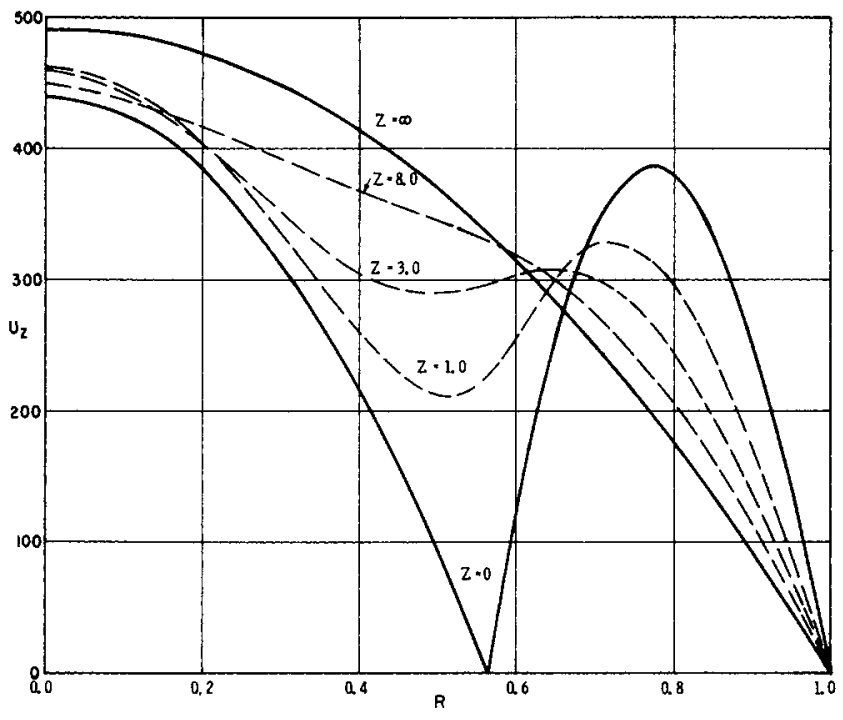

Fig. 60. Confined jet velocity profiles. $R$ un $3, \lambda=0.563$, $N_{R_{b}}=250, N_{R_{e}}=228\left(N_{R_{e}}=496\right)$.

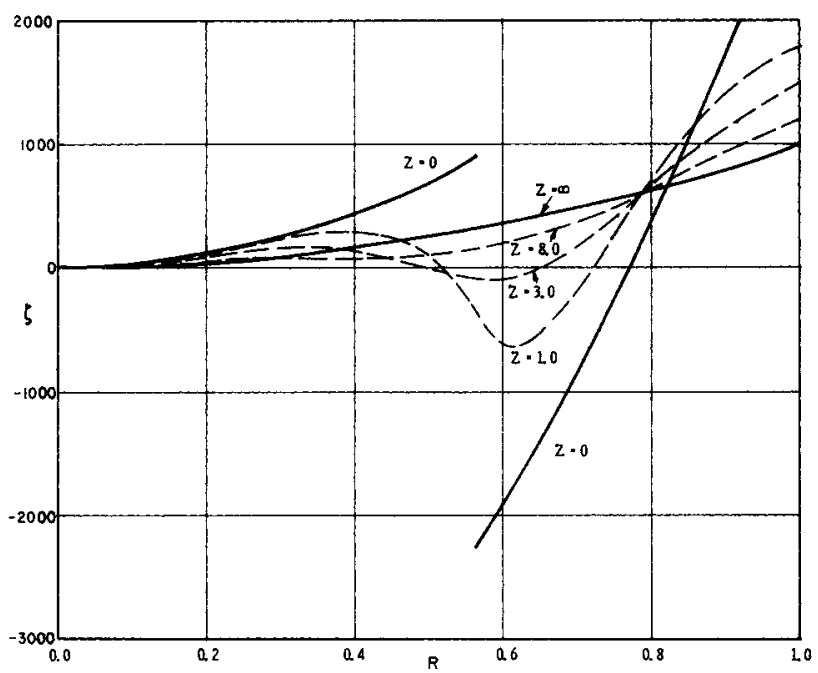

Fig. 6b. Confined iet vorticity profiles. Run $3, \lambda=0.563$, $N_{R_{e}}=250, N_{R e_{a}}=228\left(N_{R_{e}}=496\right)$.

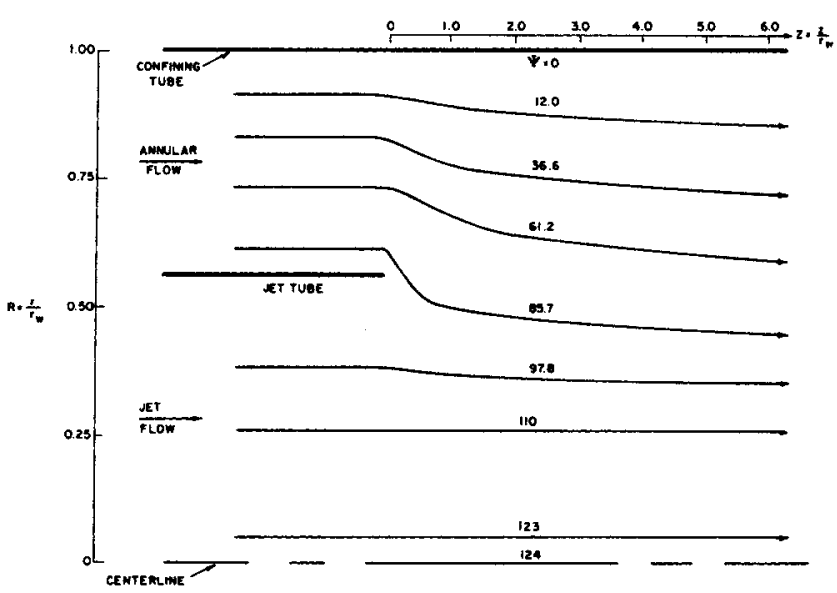

Fig. 6c. Confined jet streamlines. Run $3, \lambda=0.563, N_{R_{e}}=250$, $N_{R_{e}}=228\left(N_{R_{e}}=496\right)$. 


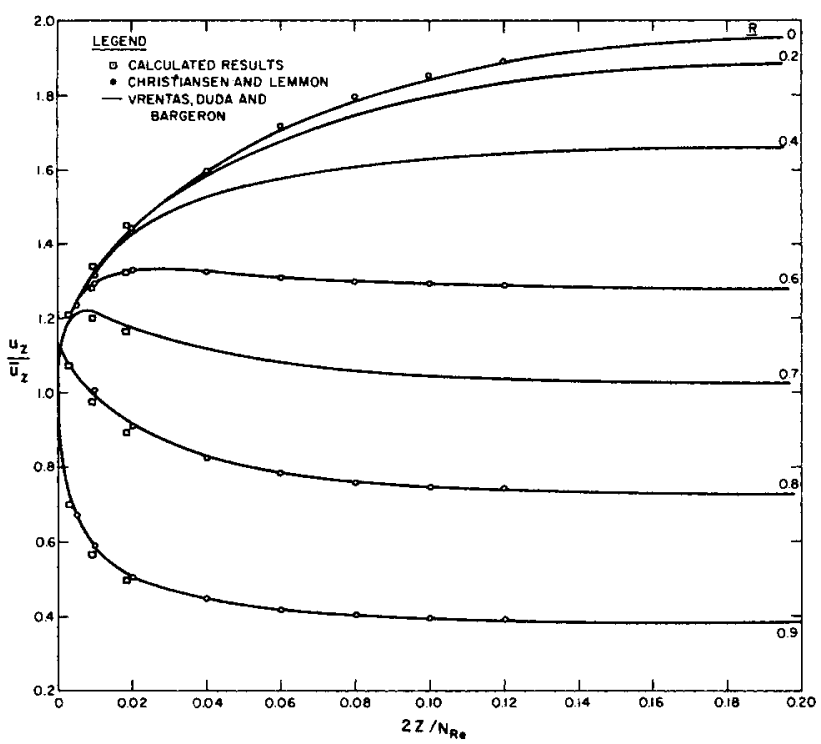

Fig. 7. Entrance region of a pipe.

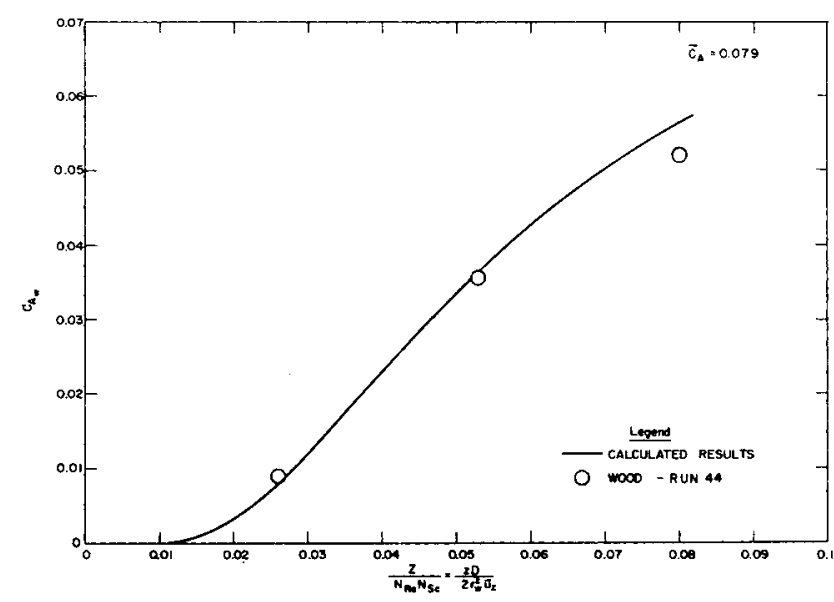

Fig. 8. Comparison of computed wall concentrations with experimental data of Wood. Run 1, $\lambda=0.281, N_{R_{b}}=139, N_{R_{e_{a}}}=354$ $\left(N_{R e}=493\right), N_{S c}=0.942$.

was adjusted to provide the same total solute flow in each of the jet streams. Clearly the wake reduced component transfer. Figure $9 a$ also indicates the sensitivity of the wall concentration to the velocity profile. Hence, the good agreement between the calculated and experimental concentrations in Figures 8 and 9 is significant.

\section{Component Transfer and Reaction}

Calculations were carried out for the gas phase reaction

$$
\mathrm{H}_{2}+\mathrm{I}_{2} \rightarrow 2 \mathrm{HI}
$$

which is essentially irreversible at $829^{\circ} \mathrm{F}$. and $1 \mathrm{~atm}$. The reaction is second order with $k=2.24 \mathrm{cu}$. ft. $/$ (lb.-mole) (sec.) during isothermal operation in a nitrogen solvent $\left(\nu \cong 7.5 \times 10^{-4} \mathrm{sq} . \mathrm{ft} . / \mathrm{sec}\right.$. $)$. For the purposes of illustration, the flow regime in run 3 (Table 2) was used, and the Schmidt numbers for $\mathrm{H}_{2}$ and $\mathrm{I}_{2}$ diffusion in nitrogen were assumed to be 0.942 at this high temperature. Inlet concentrations of $\mathrm{H}_{2}$ and $\mathrm{I}_{2}$ were both $0.013 \mathrm{lb}$.-moles/cu. $\mathrm{ft}$., $r_{w}=$ $0.33 \mathrm{ft}$., and $a=b=1$. These specifications completely characterized the confined jet reactor, since $\lambda, N_{R e_{b}}$, $N_{R e}, N_{S c}, K_{A}$, and $K_{B}$ determine the flow field and rates of conversion.

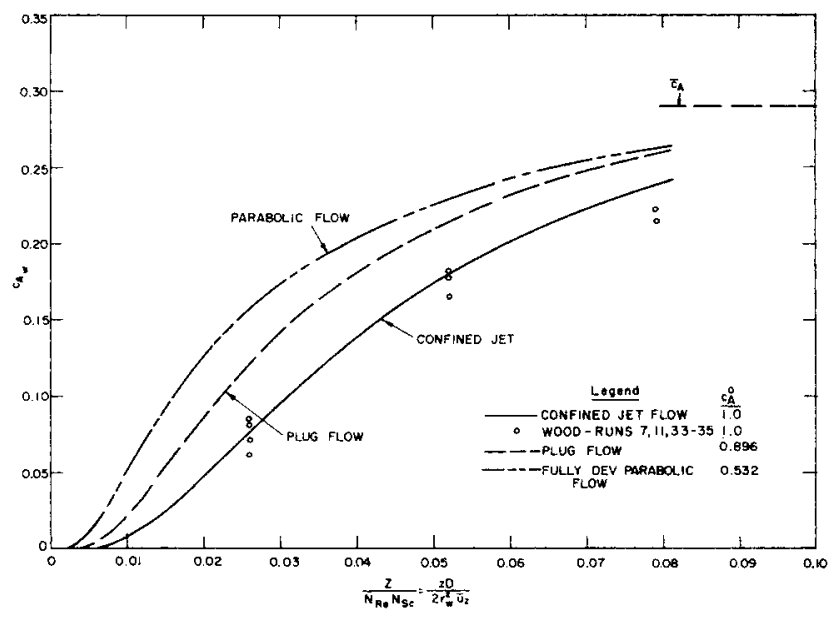

Fig. 9a. Comparison of computed wall concentrations for threeflow patterns with experimental data of Wood. Run 3, $\lambda=0.563$. $N_{R_{e}}=250, N_{R e_{a}}=228\left(N_{R e}=496\right), N_{S c}=0.942$.

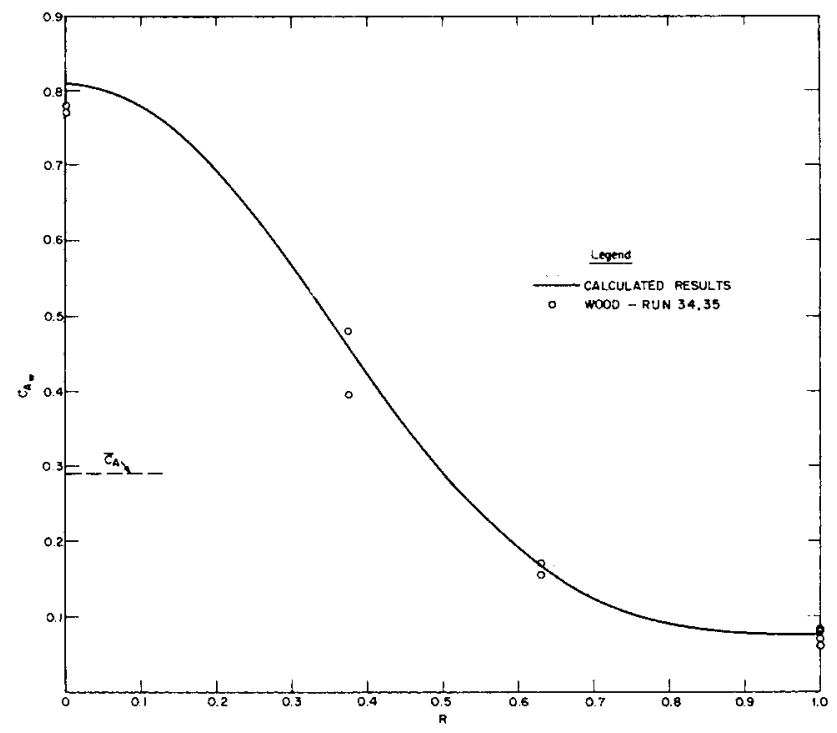

Fig. 9b. Comparison of computed concentration profile at $Z=24$ with experimental data of Wood. Run 3, $\lambda=0.563, N_{R e_{b}}=250$, $N_{R_{e}}=228\left(N_{R_{e}}=496\right), N_{5 c}=0.942$.

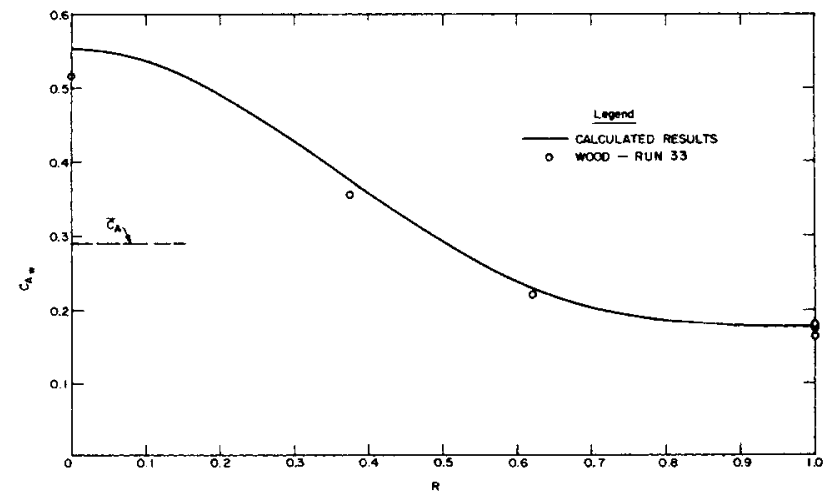

Fig. 9c. Comparison of computed concentration profile at $Z=48$ with experimental data of Wood. Run 3, $\lambda=0.563, N_{R e_{b}}=250$, $N_{R_{a}}=228\left(N_{R_{e}}=496\right), N_{S_{c}}=0.942$. 
The reaction rate in the confined jet entrance region is illustrated in Figure 10. The differential rates of conversion are plotted for the confined jet entrance region and for a plug-flow reactor with premixed feed and zero backmixing. The accumulative conversions for these two conditions are plotted in Figure 11. These plots provide an indication of the efficiency of the jet mixing.

TABLE 2. SUMmary of EXPERIMENTal Results fOR $\lambda=0.5$

\begin{tabular}{lrrrr}
\hline $\begin{array}{c}\text { Flow } \\
\text { regime }\end{array}$ & $N_{R e_{b}}$ & $N_{R e_{a}}$ & $N_{R e}$ & $\begin{array}{c}\text { Transition } \\
\text { length, in. }\end{array}$ \\
\hline Laminar & 900 & 650 & 1,400 & $>48$ \\
throughout & 1,000 & 750 & 1,600 & $>48$ \\
entrance region & 1,200 & 750 & 1,700 & $>48$ \\
Laminar & 1,200 & 980 & 2,050 & 14 \\
with turbulent & 1,200 & 580 & 1,500 & 24 \\
breakup & 1,200 & 450 & 1,300 & 22 \\
downstream & 1,200 & 350 & 1,100 & 13 \\
& 1,650 & 1,400 & 2,900 & 3 \\
& 1,650 & 1,280 & 2,750 & 7 \\
& 1,650 & 1,150 & 2,500 & 13 \\
& 1,650 & 950 & 2,250 & 15 \\
& 1,650 & 750 & 1,950 & 21 \\
& 1,650 & 550 & 1,650 & 24 \\
Turbulent & 1,650 & 450 & 1,500 & 21 \\
at jet exit & 1,650 & 400 & 1,450 & 19 \\
& 980 & 1,450 & 2,700 & 0 \\
& 980 & 1,150 & 2,200 & 0 \\
& 980 & 950 & 1,900 & 0 \\
& 1,200 & 980 & 2,050 & 0 \\
\hline
\end{tabular}

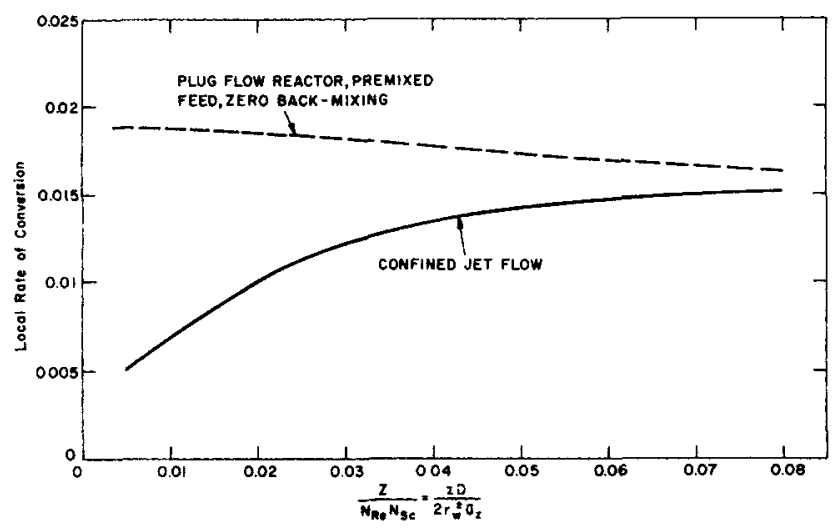

Fig. 10. Comparison of reoction rate in a confined jet reactor and a premixed plug-flow reactor. $R u n 3, \lambda=0.563, N_{R e b}=250$, $N_{R e_{a}}=228\left(N_{R_{e}}=496\right), N_{S_{c}}=0.942, K_{A}=K_{B}=4.41$.

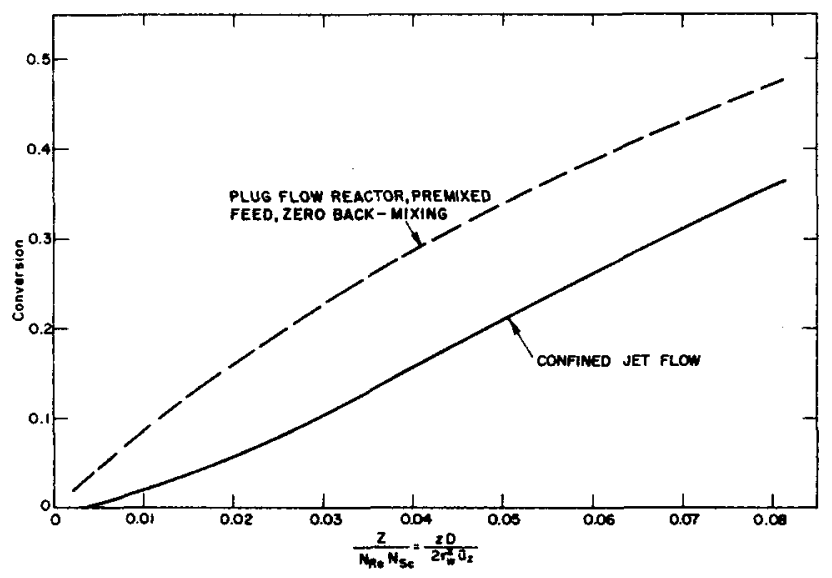

Fig. 11. Comparison of conversion in a confined jet reactor and a premixed plug-flow reactor. Run $3, \lambda=0.563, N_{R} e_{b}=250$,

$N_{R e_{a}}=228\left(N_{R_{e}}=496\right), N S_{c}=0.942, K_{A}=K_{B}=4.41$.

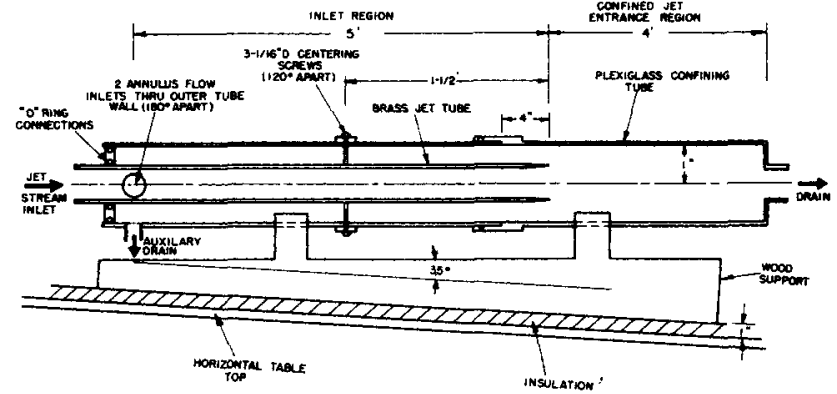

Fig. 12. Experimental apparatus.

\section{EXPERIMENTAL WORK}

The objective of the experimental work was to determine the conditions under which the laminar confined jet mixing occurred in liquids, and thereby established the range of applicability of the laminar model used for the theoretical calculations. Laminar confined jet mixing in gases has been experimentally described by Wood (12). The apparatus illustrated in Figure 12 was used for the experiments herein. An aqueous jet stream marked with tracer quantities of dye was used for flow visualization.

\section{Apparatus}

Two 55-gal. drums positioned one floor above the confined jet tubes provided smooth flow under a gravitational head. Calibrated rotorneters were used to measure flow rates of the jet and annular streams. The apparatus was positioned horizontally for visual observations but for the measurements was inclined at a 3.5-deg. angle to facilitate the removal of residual air bubbles. The system was isolated from background vibrations with flexible, shockabsorbing media. Rubber hoses were used to connect the jet tube and annulus inlets to external lines, as well as for drain lines.

The two brass jet tubes used for the experiments each extended $5 \mathrm{ft}$. into the confining tube and had the following dimensions:

\begin{tabular}{ccc} 
I.D., in. & Wall thickness, in. & O.D., in. \\
\hline$\frac{9}{16}$ & $\frac{1}{32}$ & $\frac{1}{2}$ \\
1 & $\frac{1}{16}$ & $1 \frac{1}{8}$
\end{tabular}

Both tubes were tapered inside and outside, beginning 2 in. from the jet exit to minimize instability created by vortices originating behind the leading edge of the jet tube. At the jet exit, the jet tube thickness was several hundred microns.

\section{Experimental Results}

At suffic iently low Reynolds numbers the flow remained laminar throughout the 4-ft. mixing region. A cylinder of dye was observed to exit from the jet entrance with slight contraction or expansion. Slight wavering occurred at the cylindrical surface in what appeared to be low-amplitude, sinusoidal oscillations. At higher Reynolds numbers, laminar flow was maintained for some distance downstream followed by a sudden breakup into turbulent eddies, which then dampened out a short distance further downstream. The transition distance for turbulent breakdown was a function of $N_{R e_{b}}, N_{R e_{a}}$, and the background vibration level. At high $N_{R e_{b}}$ and $N_{R e_{a}}$ turbulent eddies appeared directly behind the jet entrance.

The experimental results summarized in Table 2 for $\lambda=0.5$ indicate approximate conditions under which the various flow regimes were observed. Curves are not presented for transition lengths since these lengths were sensitive to background vibration in the laboratory even after precautions were taken to isolate the system.

Jet and annular Reynolds numbers below those in Table 2 were not investigated because meandering (unsteady, slowly wavering) flow was encountered. Mean velocities were in the order of 0.05 $\mathrm{ft} . / \mathrm{sec}$. for the experiments in Table 1.

The cylindrical structure of the jet, as indicated by the dye, was maintained in the laminar mixing region for liquids above the meandering regime, with hardly any expansion or contraction owing to low net radial flow and low diffusion rates.

\section{SUMMARY AND CONCLUSIONS}

An algorithm was successfully developed for the calculation of momentum transfer, component transfer, and chemical 
conversion in a region of confined jet mixing. The implicit, alternating-direction method was adapted to solve the nonlinear equations for the conservation of momentum with the severe boundary conditions corresponding to the confined jet entrance. The equations were converted from parabolic to elliptic form by adding unsteady state terms, although the initial conditions and transient behavior had no physical significance.

Illustrative calculations were carried out for jet mixing, jet mixing with component transfer between the streams, and jet mixing with chemical conversion and component transfer. Instability was encountered in the calculations for jet-to-annular velocity ratios greater than 4.0 , but it is presumed that stable calculations could be attained at ratios as high as 10.0 with a smaller grid size.

Computed values for the velocity field agree with the prior results of Christiansen and Lemmon and Vrentas, Duda, and Bargeron for the limiting case of developing flow in the entrance region of a pipe. Computed values for the composition agree with the experimental values of Wood for component transfer in a region of confined jet mixing. No data are available for a direct confirmation of the computed compositions for a confined jet reactor. However, these computed values provide an indication of the efficiency of such a reactor as compared to a plug-flow reactor with premixed feed.

Experimental observations indicate that a stable, laminar regime, such as that calculated herein, exists for sufficiently low Reynolds numbers. At higher Reynolds numbers turbulent breakup occurs some distance downstream from the jet entrance. At still higher Reynolds numbers the breakup occurs at the jet entrance. It was not possible to define these regimes precisely owing to a critical dependence on minor perturbations such as the level of vibration in the laboratory.

\section{ACKNOWLEDGMENT}

The assistance and services provided by the University of Michigan Computing Center are gratefully acknowledged. Financial support for Warren D. Seider and for some aspects of the work were provided by the National Aeronautics and Space Administration in the form of a Traineeship.

\section{NOTATION}

$A=$ component $A$, jet stream solute

$a=$ stoichiometric coefficient and reaction order for reactant $A$

$B=$ component $B$, annular stream solute

$b=$ stoichiometric coefficient and reaction order for reactant $B$

$C=$ dimensionless concentration, $=\mathrm{c} / \mathrm{c}^{\circ}$.

$c=$ concentration, moles/cu. $\mathrm{ft}$.

$D=$ diffusivity, sq. $\mathrm{ft} . / \mathrm{sec}$.

$j=$ radial grid point, $j=R / \Delta R$

$k=$ reaction rate constant, [moles/(cu. ft.)(sec.)] [moles/cu. ft. ] $]^{a+b}$

$K_{A}=$ dimensionless reaction rate, $=k\left(c_{A}^{o}\right)^{a-1}\left(c_{B}^{o}\right)^{b} r_{w^{\prime}}^{2} \nu$

$K_{B}=$ dimensionless reaction rate, $=\frac{b}{a} k\left(c_{A}^{o}\right)^{a}\left(c_{B}^{o}\right)^{b-1} r_{w}^{2} / \nu$

$N_{R e}=$ overall Reynolds number, $=\frac{2 r_{w} \bar{u}_{z}}{\nu}$

$N_{R e_{a}}=$ Reynolds number in annulus, $=\frac{2\left(r_{w}-r_{c}\right) \bar{u}_{z_{a}}}{\nu}$

$N_{R e_{b}}=$ Reynolds number in jet tube, $=\frac{2 r_{c} \bar{u}_{z_{b}}}{\nu}$

$N_{S c}=$ Schmidt number, $=\nu / D$

$P=$ dimensionless pressure, $=p r_{w}^{2} / \rho \nu^{2}$

$p=$ pressure, 1 b. $/(\text { sec. })^{2}(\mathrm{ft}$.
$R=$ dimensionless radius, $=r / r_{w}$

$r=$ radial distance, $\mathrm{ft}$.

$t=$ time, sec.

$U=$ dimensionless velocity component, $=u r_{w} / \nu$

$u=$ velocity component, $\mathrm{ft} . / \mathrm{sec}$.

$Z=$ dimensionless axial distance, $=z / r_{w}$

$z=$ axial distance, $\mathrm{ft}$.

\section{Greek Lefters}

$\alpha=$ dimensionless coefficient, $=\left(1-\lambda^{2}\right) / \ln \left(\frac{1}{\lambda}\right)$

$\beta=$ dimensionless coefficient, $=1+\lambda^{2}$

$\gamma_{1}=\left(c_{B}^{o} / c_{A}^{o}\right)^{b}$

$\gamma_{2}=\left(c_{B}^{O} / c_{A}^{O}\right)^{b-1}$

$\Delta \theta=$ stream function equation time step

$\Delta \tau=$ vorticity equation time step

$\Delta R=$ radial grid spacing

$\Delta Z=$ axial grid spacing

$\zeta=$ dimensionless radial vorticity, $=R\left[\frac{\partial U_{r}}{\partial Z}-\frac{\partial U_{z}}{\partial R}\right]$

$\theta=$ dimensionless 'time" in Equation (17)

$\lambda=$ dimensionless jet radius, $=r_{c} / r_{w}$

$\nu=$ kinematic viscosity, sq. ft. $/$ sec.

$\rho=$ density, lb./cu. ft.

$\tau=$ dimensionless time, $=t \nu / r_{w}^{2}$

$\Phi=\frac{C_{A}}{\gamma_{1}}-\frac{C_{B}}{\gamma_{2}}$

$\Psi=$ dimensionless stream function, $\left[d \Psi=R\left(U_{r} d Z-U_{z} d R\right)\right]$

\section{Subscripts}

$A=$ component $A$

$a=$ annular stream

$B=$ component $B$

$b=$ central jet stream

$c=$ jet (inner) tube

$i=$ grid point whose axial coordinate is $Z=i \cdot \Delta Z$

$j=$ grid point whose radial coordinate is $R=j \cdot \Delta R$

$L=$ entrance length to attain $99 \%$ fully developed flow

$m=i$ at $Z_{L}$

$n=$ at the confining tube wall, $j=n$

$r=$ radial direction

$w=$ confining (outside) tube

$z=$ axial direction

\section{Superscripts}

$o=$ inlet

$-=$ average

* = value at the end of the half-time step $\Delta \tau / 2$

' = value at the end of the time step $\Delta \tau$

\section{LITERATURE CITED}

1. Hellums, J. D., and S. W. Churchill, "International Developments in Heat Transfer," Pt. V, p. 118, Am. Soc. Mech. Engrs., New York (1961).

2.

3. Wilkes, J. O., and S. W. Churchill, ibid., 12, 161 (1966).

4. Sa muels, M. R., and S. W. Churchill, ibid., 13, 77 (1967).

5. Seider, W. D., Ph.D. thes is, Univ. Michigan, Ann Arbor (1966).

6. Curtet, R., Combustion Flame, 2, 383 (1958).

7. Wang, Y. L., and P. A. Longwell, AlChE J., 10, 323 (1964).

8. Vrentas, J. S., J. L. Duda, and K. G. Bargeron, ibid., 12, 837 (1966).

9. Paris, J., and S. Whitaker, ibid., 11, 1033 (1965).

10. Burke, S. P., and T. E. W. Schumann, Ind. Eng. Chem., 20, 998 (1928).

11. Savage, L. D., Combustion Flame, 6, 77 (1962).

12. Wood, B., D. Sc. thes is, Massachusetts Inst. Technol., Cambridge (1964).

13. Christiansen, E. B., and H. E. Lemmon, AlChE J., 11, 995 (1965).

Manuscript received December $26 ; 1968$; revision received July 14,1969; paper accepted July 18,1969. Paper presented at AIChE LOS Angeles meeting. 